Maria Esther de Carvalho 1

Rubens Antonio da Silva 1

Vera Lúcia Cortiço Corrêa Rodrigues 2

Cleide Dantas de Oliveira 3

\section{Programa de Controle da Doença de Chagas no Estado de São Paulo: sorologia de moradores como parte de investigação de unidades domiciliares com presença de triatomíneos vetores na década de 1990}

\author{
The Chagas Disease Control Program of the \\ São Paulo State: the contribution of serology \\ to the epidemiological investigation of triatomine- \\ infested domiciliary units during the 1990s
}

1 Laboratório de Soroepidemiologia Superintendência de Controle de Endemias. Rua Paula Souza 166, São Paulo, SP 01027-000, Brasil. esther@sucen.sp.gov.br rubens@sucen.sp.gov.br

2 Laboratório de Parasitoses por Flagelados, Superintendência de Controle de Endemias. Rua Afonso Pessini 86, C. P. 192, Mogi-Guaçu, SP 13840-970, Brasil. verana@dglnet.com.br

3 Serviço Regional de São Vicente, Superintendência de Controle de Endemias. Rua João Ramalho 587, São Vicente, SP 11310-050, Brasil. sr02@sucen.sp.gov.br

\begin{abstract}
The Chagas Disease Control Program in São Paulo State, Brazil, now in the entomological surveillance phase, includes a serological examination of individuals residing in domiciliary units infested with vector triatomines infected with Trypanosoma cruzi. From 1990 to 1999, this action included area in which triatomine searches were conducted either as a routine procedure, according to their levels of intra- or peridomiciliary infestation, or at the request of local residents. Among residents of the 1,415 UDs inspected, we collected 5,587 blood samples for serological examination, 87 of which (1.56\%) tested positive, seven of which from individuals under 29 years of age. The species most frequently captured were Panstrongylus megistus, Triatoma sordida, and Triatoma tibiamaculata in the adult stage. No association was found between presence of seropositive residents and triatomines infected with $\mathrm{Tr}$. cruzi $(O R=1.498$; $0.875<O R<2.564,95 \%$ C.I.). Our purpose was to use serological testing to investigate the situation of areas identifiable as being at risk of Chagas disease transmission and to compare the results with extant data about Tr. cruzi infection both in humans and vector triatomines.
\end{abstract}

Key words Chagas Disease; Surveillance; Triatominae

Resumo O Programa de Controle da Doença de Chagas (PCDCh) no Estado de São Paulo encontrase na fase de vigilância entomológica, submetendo a exames sorológicos os moradores de unidades domiciliares (UDs), em que triatomíneos vetores tenham sido encontrados infectados por Trypanosoma cruzi. No decênio 1990-1999 foram trabalhadas localidades que, segundo graus de infestações no intra ou no peridomicílio, desencadearam trabalhos de rotina de busca desses vetores e de atendimento a notificações por parte de moradores. Em 1.415 UDs examinadas, 5.587 amostras de sangue foram obtidas, 87 (1,56\%) das quais reagentes. Dessas, sete correspondiam a pessoas com menos de 29 anos de idade. As espécies mais freqüentemente associadas, em suas formas adultas, às UDs foram: Panstrongylus megistus, Triatoma sordida $e$ T. tibiamaculata. Não foi observada associação entre UDs com sororreagentes e presença de triatomíneos infectados por Tr. cruzi (odds ratio $=1,498 ; 0,875<O R<2,564,95 \%$ de confiança). Propõe-se utilizar inquéritos sorológicos amostrais no PCDCh, para investigar a situação prevalente em áreas identificáveis como de risco de transmissão, complementando-os com estudos isolados de Tr. cruzi aí obtidos.

Palavras-chave Doença de Chagas; Vigilância; Triatominae 


\section{Introdução}

A relevância do uso da sorologia em todas as fases de uma campanha contra a doença de Chagas: preparatória, ataque e consolidação, foi destacada por Hoff et al. (1985). Inquéritos abrangentes permitem detectar variações de comportamento sorológico ao longo de diversas faixas etárias (Albarracin-Veizaga et al., 1999; Carvalho et al., 1987, 1993/1994; Costa et al., 1982; Coutinho, 1962; Salvatella et al., 1989; Solís-Acosta et al., 1997; Velasco-Castrejón et al., 1992). Verificar proporções de soropositividade por faixa etária e sexo, contribui para a análise de características da transmissão, associadas ou não a medidas de controle de vetores. Crianças nascidas após a implantação de tais medidas representam indicadores de sua eficácia (Andrade, 1994; Andrade et al., 1992; Chuit et al., 1989; Dias, 1967; Goldsmith et al., 1985; Hoff et al., 1985; Rocha-e-Silva et al., 1979). O conhecimento prévio da situação epidemiológica permite avaliar riscos potenciais de transmissão em grupos específicos (Andrade, 1994; Piesman et al., 1985; Velasco-Hernández, 1991).

No Estado de São Paulo, Brasil, a Superintendência de Controle de Endemias (SUCEN) optou pela aplicação de inquéritos sorológicos em crianças, após as expressivas medidas de combate aos vetores, realizadas no período entre 1951 e 1967. Em fins da década de 1960, foram amostrados escolares de 9 a 14 anos, da rede oficial de ensino, em todos os municípios do Estado, à exceção dos da Grande São Paulo (Guarita et al., 1978). Nos 54 municípios que tinham apresentado as mais altas prevalências, deu-se prosseguimento a inquéritos entre alunos da primeira série do primeiro grau de escolas de zona rural, seguidamente entre os anos de 1973 e 1983 (Carvalho, 2000). No final de 1983, avaliado por uma Comissão Interna de Técnicos da SUCEN, o Programa de Controle da Doença de Chagas (PCDCh), encerra essa atividade e passa a utilizar a sorologia aplicada a moradores de Unidades Domiciliares (UDs), notificantes de triatomíneos ou daquelas em que a detecção dos vetores decorreu de atividades de rotina de busca (Carvalho, 2000).

Na fase iniciada em 1984, de consolidação/ vigilância entomológica, a obtenção da amostra de sangue para reações sorológicas era condicionada à presença, nas UDs, de: (1) triatomíneos pertencentes à espécie Triatoma infestans ou (2) triatomíneos pertencentes a outras espécies vetoras, estando positivos para Trypanosoma cruzi ou ainda ter sugado sangue humano (Buralli, 1985). A partir de 1994, houve nova mudança de critérios para o uso da sorologia as- sociada à doença de Chagas, ou seja, necessidade de demonstrar a existência, no intradomicílio, de triatomíneos infectados por Tr. cruzi.

Diante da ausência de capturas de exemplares de T. infestans em atividades de pesquisa rotineira de triatomíneos e atendimentos às notificações encaminhadas pela população, nova proposta metodológica foi implantada em 1990. Tendo-se em conta infestações intra e peridomiciliares, as localidades do Estado de São Paulo, onde se inseriam as UDs a pesquisar, foram classificadas em prioridades, levando-se em consideração o cálculo das razões entre o total de intradomicílios ou peridomicílios positivos e o total de intradomicílios ou peridomicílios pesquisados. Assim, localidades com infestações intradomiciliar $\geq 5 \%$ e peridomiciliar $\geq 10 \%$ foram classificadas como Prioridade 1; localidades com infestações intradomiciliar $<5 \%$ e peridomiciliar $<10 \%$, Prioridade 2 e com infestações intradomiciliar e peridomiciliar $=$ zero, Prioridade 3 . Além de estímulo às notificações e seu atendimento, foram preconizadas, para as localidades em Prioridades 1 e 2, pesquisas nas casas e seus anexos, sendo que, nas localidades em Prioridade 2 eram amostradas aquelas a serem submetidas à pesquisa. Nas de Prioridade 3 , foi abolida a pesquisa de rotina. Incentivaram-se as notificações de triatomíneos e seu pronto atendimento, tendo em vista detectar colônias iniciais de vetores nos domicílios, metodologia já indicada para áreas de vigilância epidemiológica desde fins da década de 1960 (Carvalho, 2000).

Neste trabalho analisamos o período de 1990 a 1999, durante o qual, diante da inexistência de capturas de exemplares de T. infestans em atividades de rotina e de atendimento a notificações, alterações nos critérios de pesquisa foram adotadas.

\section{Material e métodos}

Os dados de campo e de laboratório usados nesta análise foram extraídos de arquivos da SUCEN, a partir de boletins da Divisão de Orientação Técnica (DOT), dos Serviços Regionais (SRs: 02-São Vicente, 03-Taubaté, 04-Sorocaba, 05Campinas, 06-Ribeirão Preto, 08-São José do Rio Preto, 09-Araçatuba, 10-Presidente Prudente e 11-Marília) e dos Laboratórios de Parasitoses por Flagelados de Mogi Guaçu e de Soroepidemiologia. Foram armazenados em bancos de dados Foxpro for Windows, com as variáveis: ano; serviço regional; município; localidade; número da casa; idade, sexo e resultado de exame sorológico de cada morador; espécie de triatomíneo cap- 
turada: local de captura, fase evolutiva, presença de infecção por Tr. cruzi e detecção de sangue humano em ingesta. Análises de freqüências foram obtidas por meio de programa Epinfo, versão 6.0 (Dean et al., 1994), sendo trabalhadas 1.415 UDs distribuídas em localidades de 184 municípios do estado.

De casos reagentes sorológicos foram vistos boletins SUCEN de investigação epidemiológica, dos quais se destacam as variáveis: local de nascimento, tempo de moradia no local e práticas alimentares relacionadas com a caça de animais silvestres e sua manipulação. A metodologia sorológica adotada para o processamento das amostras colhidas em papel-filtro, e submetidas à reação de imunofluorescência indireta, seguiu as prescrições de Carvalho (2000).

Com a finalidade de verificar associação entre UD com morador sororreagente e presença de triatomíneos infectados por Tr. cruzi, aplicou-se a prova odds ratio (OR), com 95\% de confiança (Dawson-Saunders \& Trapp, 1994).

\section{Resultados}

No período de 1990 a 1999, considerando-se as condições do PCDCh para executar exames sorológicos de moradores, 1.415 UDs foram trabalhadas. Presença de T. infestans de maneira isolada, oriunda de transporte passivo a partir de outras Unidades da Federação foi observada em dois municípios. Entre 1998 e 1999, foi encontrada essa espécie no Município de Paulínia, localidade Fazenda Paraíso (Leite et al., 2001), disto resultando trabalho em 9 UDs (0,64\%). Nas demais observaram-se: triatomíneos infectados em 332 UDs (23,46\%); triatomíneos infectados e com ingesta identificada como sangue humano em 54 UDs $(3,82 \%)$ e triatomíneos com ingesta identificada como sangue humano em 384 UDs $(27,14 \%)$. A obtenção de sangue de moradores em 372 UDs $(26,29 \%)$ tornou-se desnecessária por não terem sido, nessa ocasião, seguidas as normas do PCDCh. No caso de 273 UDs $(19,29 \%)$, faltaram informações sobre espécie de triatomíneo observada, sua forma evolutiva, eventual infecção por Tr. cruzi e resultado de exame de ingesta.

A Tabela 1 mostra o número de UDs trabalhadas no decênio. O declínio, mais acentuado a partir do ano de 1994, é reflexo das alterações adotadas nos critérios de inserção da sorologia no PCDCh. Das 5.587 amostras de sangue examinadas, 87 (1,56\%) apresentaram-se reagentes para anticorpos IgG humanos anti Tr. cruzi.

A distribuição dos sororreagentes por idade e sexo está apresentada na Tabela 2. Em nível de significância de $95 \%$ verifica-se que não houve diferença de positividade entre os sexos.

Quanto à faixa etária, observa-se um aumento de positividade a partir do grupo 10-14 anos. Salienta-se que dentre as mulheres sororreagentes, 25 (55,5\%) encontravam-se em idade fértil. Dos 87 casos sororreagentes, 40 (45,98\%) foram investigados. Sete casos com idade abaixo de 29 anos foram detectados $(8,05 \%$ dos positivos, $0,13 \%$ do total); dois desses investigados, com tempo de moradia na área de 1 e 27 anos, respectivamente (Tabela 3).

Tabela 1

Programa de Controle da Doença de Chagas (PCDCh): variação anual da sororreatividade para infecção chagásica nos municípios do Estado de São Paulo, Brasil, 1990 a 1999.

\begin{tabular}{|c|c|c|c|c|c|}
\hline \multirow[t]{2}{*}{ Ano } & \multirow[t]{2}{*}{ UDs examinadas } & \multirow{2}{*}{$\begin{array}{l}\text { Amostras } \\
\text { examinadas }\end{array}$} & \multicolumn{2}{|c|}{ Reagentes } & \multirow[t]{2}{*}{ IC $95 \%$} \\
\hline & & & $\mathrm{n}$ & $\%$ & \\
\hline 1990 & 246 & 993 & 13 & 1,31 & $0,767-2,227$ \\
\hline 1991 & 274 & 1.058 & 19 & 1,80 & $1,153-2,788$ \\
\hline 1992 & 257 & 929 & 7 & 0,75 & $0,366-1,547$ \\
\hline 1993 & 270 & 1.048 & 13 & 1,24 & $0,726-2,111$ \\
\hline 1994 & 134 & 517 & 11 & 2,13 & $1,192-3,769$ \\
\hline 1995 & 78 & 299 & 5 & 1,67 & $0,716-3,854$ \\
\hline 1996 & 35 & 156 & 2 & 1,28 & $0,352-4,554$ \\
\hline 1997 & 40 & 191 & 7 & 3,66 & $1,786-7,371$ \\
\hline 1998 & 18 & 71 & 3 & 4,22 & $1,447-11,703$ \\
\hline 1999 & 63 & 325 & 7 & 2,15 & $1,047-4,378$ \\
\hline Total & 1.415 & 5.587 & 87 & 1,56 & $1,264-1,917$ \\
\hline
\end{tabular}

UDs = unidades domiciliares; IC 95\% = intervalo de confiança a 95\%. 
Programa de Controle da Doença de Chagas (PCDCh): sorologia para infecção chagásica, por grupo etário e sexo, em população de municípios do Estado de São Paulo, Brasil, 1990 a 1999.

\begin{tabular}{|c|c|c|c|c|c|c|c|c|c|}
\hline \multirow[t]{3}{*}{ Grupo etário (anos) } & \multicolumn{3}{|c|}{ Feminino } & \multicolumn{3}{|c|}{ Masculino } & \multicolumn{3}{|c|}{ Total } \\
\hline & \multirow[t]{2}{*}{ Examinados } & \multicolumn{2}{|c|}{ Reagentes } & \multirow[t]{2}{*}{ Examinados } & \multicolumn{2}{|c|}{ Reagentes } & \multirow[t]{2}{*}{ Examinados } & \multicolumn{2}{|c|}{ Reagentes } \\
\hline & & $\mathrm{n}$ & $\%$ & & $\mathrm{n}$ & $\%$ & & $\mathrm{n}$ & $\%$ \\
\hline Ignorado & 1 & 0 & 0,00 & 13 & 0 & 0,00 & 14 & 0 & 0,00 \\
\hline$<1$ & 18 & 0 & 0,00 & 18 & 0 & 0,00 & 36 & 0 & 0,00 \\
\hline $1-4$ & 244 & 0 & 0,00 & 247 & 0 & 0,00 & 491 & 0 & 0,00 \\
\hline $5-9$ & 369 & 0 & 0,00 & 410 & 0 & 0,00 & 779 & 0 & 0,00 \\
\hline $10-14$ & 404 & 2 & 0,50 & 357 & 1 & 0,28 & 761 & 3 & 0,39 \\
\hline $15-19$ & 250 & 1 & 0,40 & 288 & 0 & 0,00 & 538 & 1 & 0,19 \\
\hline $20-29$ & 432 & 1 & 0,23 & 354 & 2 & 0,56 & 786 & 3 & 0,38 \\
\hline $30-39$ & 410 & 6 & 1,46 & 349 & 6 & 1,72 & 759 & 12 & 1,58 \\
\hline $40-49$ & 284 & 17 & 5,99 & 302 & 9 & 2,98 & 586 & 26 & 4,44 \\
\hline $50-59$ & 216 & 9 & 4,17 & 209 & 9 & 4,31 & 425 & 18 & 4,24 \\
\hline $60-69$ & 109 & 5 & 4,59 & 159 & 8 & 5,03 & 268 & 13 & 4,85 \\
\hline$\geq 70$ & 67 & 4 & 5,97 & 77 & 7 & 9,09 & 144 & 11 & 7,64 \\
\hline Total & 2.804 & 45 & 1,60 & 2.770 & 42 & 1,52 & 5.587 & 87 & 1,56 \\
\hline
\end{tabular}

Das 1.415 UDs trabalhadas, 70 (4,95\%) apresentaram moradores com resultado sorológico positivo para Tr. cruzi. Quando comparadas as características associadas ao vetor, nas UDs com presença ou ausência de sororreagentes, observa-se que a espécie predominante nas duas situações foi Panstrongylus megistus, seguida de T. sordida e T. tibiamaculata (Tabela 4). Nas UDs com sororreagentes houve uma diferença estatisticamente significante em nível de $95 \%$ $(\mathrm{p}<0,05)$ quanto à freqüência de T. sordida. Fases evolutivas ninfais, isoladamente calculadas sobre o total de UDs, estiveram presentes em 98 $(6,92 \%)$ dos casos e, associadas às formas adultas, representaram infestação de 7,21\% (102 UDs). Formas adultas de triatomíneos foram encontradas em 1.073 UDs (75,83\%). Em 142 UDs $(10,03 \%)$ houve perda de informação quanto à fase evolutiva dos triatomíneos encontrados.

Local de captura interno predominou sobre o externo e ingesta de sangue humano foi observada em maior proporção nos exemplares capturados nas UDs com presença de reagentes sorológicos, ainda que de forma estatisticamente não significante. Esses dados corroboram os observados na Tabela 5, que não evidenciam associação entre Unidades Domiciliares com reagentes sorológicos e presença de triatomíneos infectados por Tr. cruzi ante o valor de OR = $1,498(0,875<$ OR $<2,564,95 \%$ de confiança $)$.

\section{Discussão}

Considerando-se o risco representado pela presença de triatomíneos vetores em domicílios, descobertos durante atividades de pesquisa rotineira ou de atendimento a notificações, o PCDCh preconizou a identificação de seus hábitos alimentares e a execução de provas para o sorodiagnóstico de infecção chagásica em moradores das UDs em questão. A constatação da ausência de pessoas infectadas, nascidas após a época considerada como limite para a aquisição da doença de Chagas autóctone, tende a reforçar a idéia de ter sido interrompida a transmissão vetorial no Estado de São Paulo, e a confirmar a hipótese aceita para a fixação dessa ocasião entre fins da década de 1960 e início da de 1970 (Buralli, 1985). Assim originou-se o uso da sorologia no Programa. Seguindo esse raciocínio, neste trabalho, teoricamente, não seria de se esperar a ocorrência de pessoas sororreagentes nascidas após 1970, como observado em sete casos de idade inferior a 29 anos. Como foram incompletas, não só as informações relacionadas a tais casos, como também aos de idades maiores, não se pode deduzir qual tenha sido o modo de transmissão. É provável que a ausência de investigação de casos tenha decorrido da falta de infra-estrutura de apoio ao infectado chagásico. Outra hipótese para explicar a não realização dessas investigações seria a de o PCDCh, naquele momento, ocupar um plano não prioritário dentre as demais atividades da 
Programa de Controle da Doença de Chagas (PCDCh): caracterização de casos sororreagentes para infecção chagásica nos municípios do Estado de São Paulo, Brasil, 1990 a 1999.

\begin{tabular}{|c|c|c|c|c|c|c|}
\hline Ano* & SR & Município/Localidade* & $\begin{array}{l}\text { Idade } \\
\text { (anos) }\end{array}$ & Sexo & Naturalidade & $\begin{array}{l}\text { Tempo } \\
\text { de residência** }\end{array}$ \\
\hline 1990 & 02 & Miracatu/Bairro Barra Funda & 33 & M & Itaipe, Minas Gerais & 16 anos \\
\hline 1990 & 02 & Juquiá/Bairro Iporanga & 41 & $\mathrm{~F}$ & Mundo Novo, Bahia & 15 anos \\
\hline 1990 & 06 & Jardinópolis/Rua Getúlio Vargas & 67 & $\mathrm{~F}$ & Caconde, São Paulo & $4 \operatorname{anos}^{\star \star \star}$ \\
\hline 1991 & 02 & Juquiá/Bairro Corujas & 14 & $\mathrm{~F}$ & Paulistana, Piauí & 1 ano \\
\hline 1991 & 05 & Caconde/Localidade 31 & 63 & M & Caconde, São Paulo & 63 anos \\
\hline 1991 & 05 & Caconde/Fazenda Bom Jesus & 64 & $\mathrm{~F}$ & Caconde, São Paulo & 64 anos \\
\hline 1991 & 05 & Divinolândia/Fazenda Santa Virgínia & 50 & $\mathrm{~F}$ & Divinolândia, São Paulo & 50 anos \\
\hline 1991 & 05 & Divinolândia/Bairro Sertãozinho & 63 & $\mathrm{~F}$ & Divinolândia, São Paulo & 63 anos \\
\hline 1992 & 02 & Registro/Bairro Guaviruva (Margem Esquerda) & 37 & M & Teófilo Otoni, Minas Gerais & 16 anos \\
\hline 1993 & 02 & Peruíbe/Jardim Caraguava & 49 & $\mathrm{~F}$ & Teófilo Otoni, Minas Gerais & 36 anos \\
\hline 1993 & 04 & Itaberá/Bairro Engenheiro Maia & 49 & M & Barão de Antonina, São Paulo & 49 anos $^{\star \star \star}$ \\
\hline 1993 & 04 & Coronel Macedo/Bairro Barra Grande & 66 & M & Itaporanga, São Paulo & $66 \operatorname{anos}^{\star \star \star}$ \\
\hline 1993 & 04 & Sorocaba/Bairro Aleixos & 75 & M & Angatuba, São Paulo & 75 anos $^{\star \star \star}$ \\
\hline 1993 & 04 & Itararé/Bairro Matão & 45 & M & Itararé, São Paulo & 45 anos \\
\hline 1993 & 04 & Itaporanga/Bairro São Sebastião da Barra Grande & 45 & $\mathrm{~F}$ & Taquarituba, São Paulo & 45 anos $^{\star \star \star}$ \\
\hline 1993 & 04 & Itaporanga/Bairro do Desejo & 50 & $\mathrm{~F}$ & Itaporanga, São Paulo & 50 anos $^{\star \star \star}$ \\
\hline 1993 & 04 & Itaberá/Bairro Limeira & 39 & $\mathrm{~F}$ & Itaberá, São Paulo & 39 anos \\
\hline 1993 & 11 & Promissão/Vila Dionísia IV & 57 & $\mathrm{~F}$ & Cafelândia, São Paulo & $8 \operatorname{anos}^{\star \star *}$ \\
\hline 1993 & 11 & Promissão/Bairro Lagoa Bonita & 38 & $\mathrm{~F}$ & São Paulo, São Paulo & 15 anos $^{\star \star \star}$ \\
\hline 1993 & 11 & Promissão/Bairro Lagoa Bonita & 33 & M & Promissão, São Paulo & 33 anos \\
\hline 1993 & 11 & Santa Cruz do Rio Pardo/Bairro Figueira Branca & 41 & $\mathrm{~F}$ & Óleo, São Paulo & 41 anos \\
\hline 1994 & 02 & Registro/Bairro Guaviruva (Margem Esquerda) & 39 & $\mathrm{~F}$ & Teófilo Otoni, Minas Gerais & 1 ano \\
\hline 1994 & 02 & Registro/Bairro Guaviruva (Margem Esquerda) & 45 & M & Teófilo Otoni, Minas Gerais & 1 ano \\
\hline 1994 & 05 & Socorro/Bairro Pereiras & 55 & M & Pernambuco & 13 anos \\
\hline 1995 & 02 & Miracatu/Localidade Faú & 33 & M & Nova Cruzeiro, Minas Gerais & 11 anos \\
\hline 1995 & 02 & Pariquera-Açu/Bairro 13 de Maio & 77 & M & São Gotardo, Minas Gerais & 1 ano \\
\hline 1995 & 05 & São João da Boa Vista/Bairro Capituva & 67 & $\mathrm{~F}$ & São João da Boa Vista, São Paulo & 67 anos \\
\hline 1995 & 09 & General Salgado/Fazenda São José & 27 & M & General Salgado, São Paulo & 27 anos \\
\hline 1996 & 02 & Jacupiranga/Fazenda Lageado & 52 & M & Muritiba, Bahia & 15 anos \\
\hline 1996 & 02 & Bertioga/Boracéia & 60 & M & Bahia & 45 anos \\
\hline 1997 & 02 & Pariquera-Açu/Vila Roseli & 55 & $\mathrm{~F}$ & Avaré, São Paulo & Ignorado \\
\hline 1997 & 02 & Pariquera-Açu/Vila Roseli & 60 & $\mathrm{M}$ & Avaré, São Paulo & Ignorado \\
\hline 1998 & 02 & Juquiá/Fazenda Iporanga & 44 & $\mathrm{M}$ & Sete Barras, São Paulo & Ignorado \\
\hline 1998 & 08 & São José do Rio Preto/Monteiro Lobato & 81 & M & Jardinópolis, São Paulo & 18 anos \\
\hline 1999 & 05 & Paulínia/Fazenda Paraíso & 45 & $\mathrm{~F}$ & Carmem do Rio Claro, Minas Gerais & Ignorado \\
\hline 1999 & 06 & Colina/Fazenda Baixadinha & 60 & M & Guaraci, São Paulo & 2 anos \\
\hline 1999 & 06 & Colina/Fazenda Baixadinha & 58 & $\mathrm{~F}$ & Bahia & 2 anos \\
\hline 1999 & 08 & Guapiaçu/Rua Antônio Hernandes & 36 & $\mathrm{M}$ & Palestina, São Paulo & 14 anos \\
\hline 1999 & 08 & Guapiaçu/Rua Antônio Hernandes & 75 & $\mathrm{~F}$ & Salinas, Minas Gerais & 20 anos \\
\hline 1999 & 08 & Floreal/Bairro Gariroba I & 48 & $\mathrm{~F}$ & Monções, São Paulo & 6 anos \\
\hline
\end{tabular}

$\mathrm{SR}=$ Serviço Regional da Superintendência de Controle de Endemias:

* Ano, município e localidade de detecção do caso;

** Tempo de residência no local de detecção do caso:

*** Tempo de moradia no local de detecção e em municípios vizinhos de passado endêmico para doença de Chagas no Estado. 
Programa de Controle da Doença de Chagas (PCDCh): unidades domiciliares (UDs) com e sem presença de sororreagentes para infecção chagásica, de acordo com características associadas ao vetor, em municípios do Estado de São Paulo, Brasil, 1990 a 1999.

\begin{tabular}{|c|c|c|c|c|c|c|c|c|c|}
\hline \multirow[t]{2}{*}{ Característica } & \multicolumn{3}{|c|}{$\begin{array}{l}\text { UD com sororreagente } \\
\qquad(\mathrm{n}=70)\end{array}$} & \multicolumn{3}{|c|}{$\begin{array}{l}\text { UD sem sororreagentes } \\
\qquad(n=1.345)\end{array}$} & \multicolumn{3}{|c|}{ Total $(n=1.415)$} \\
\hline & $\mathrm{n}$ & $\%$ & $\% \star$ & $\mathrm{n}$ & $\%$ & $\% \star$ & $\mathrm{n}$ & $\%$ & $\%$ * \\
\hline \multicolumn{10}{|l|}{ Espécie ** } \\
\hline T. infestans & 0 & 0,0 & 0,0 & 1 & 0,1 & 0,1 & 1 & 0,1 & 0,1 \\
\hline P. megistus & 27 & 38,6 & 46,6 & 605 & 45,0 & 49,7 & 632 & 44,7 & 49,5 \\
\hline T. sordida & 21 & 30,0 & 36,2 & 250 & 18,6 & 20,5 & 271 & 19,1 & 21,2 \\
\hline R. neglectus & 4 & 5,7 & 6,9 & 43 & 3,2 & 3,5 & 47 & 3,3 & 3,7 \\
\hline T. tibiamaculata & 4 & 5,7 & 6,9 & 263 & 19,6 & 21,6 & 267 & 18,9 & 20,9 \\
\hline T. arthurneivai & 0 & 0,0 & 0,0 & 1 & 0,1 & 0,1 & 1 & 0,1 & 0,1 \\
\hline R. domesticus & 0 & 0,0 & 0,0 & 28 & 2,1 & 2,3 & 28 & 1,9 & 2,2 \\
\hline P. megistus + T. tibiamaculata & 0 & 0,0 & 0,0 & 18 & 1,3 & 1,5 & 18 & 1,3 & 1,4 \\
\hline$T$. sordida $+R$. neglectus & 2 & 2,9 & 3,4 & 2 & 0,1 & 0,2 & 4 & 0,3 & 0,3 \\
\hline$P$. megistus $+R$. domesticus & 0 & 0,0 & 0,0 & 3 & 0,2 & 0,2 & 3 & 0,2 & 0,2 \\
\hline Outros & 0 & 0,0 & 0,0 & 4 & 0,3 & 0,3 & 4 & 0,3 & 0,3 \\
\hline Sem informação & 12 & 17,1 & & 127 & 9,4 & & 139 & 9,8 & \\
\hline \multicolumn{10}{|l|}{ Fase evolutiva e infecção } \\
\hline Ninfas & 4 & 5,7 & 6,8 & 56 & 4,2 & 4,6 & 60 & 4,2 & 4,7 \\
\hline Ninfas infectadas & 4 & 5,7 & 6,8 & 34 & 2,5 & 2,8 & 38 & 2,7 & 3,0 \\
\hline Ninfas e adultos & 3 & 4,3 & 5,1 & 56 & 4,2 & 4,6 & 59 & 4,2 & 4,6 \\
\hline Ninfas e adultos infectados & 9 & 12,9 & 15,3 & 34 & 2,5 & 2,8 & 43 & 3,0 & 3,4 \\
\hline Adultos & 29 & 41,4 & 49,1 & 739 & 54,9 & 60,9 & 768 & 54,3 & 60,3 \\
\hline Adultos infectados & 10 & 14,3 & 16,9 & 295 & 21,9 & 24,3 & 305 & 21,6 & 24,0 \\
\hline Sem informação & 11 & 15,7 & & 131 & 9,7 & & 142 & 10,0 & \\
\hline \multicolumn{10}{|l|}{ Local de captura } \\
\hline Interno & 41 & 58,6 & 71,9 & 1049 & 78,0 & 87,6 & 1090 & 77,0 & 86,8 \\
\hline Externo & 11 & 15,7 & 19,3 & 117 & 8,7 & 9,8 & 128 & 9,1 & 10,2 \\
\hline Interno e externo & 5 & 7,1 & 8,8 & 32 & 2,4 & 2,6 & 37 & 2,6 & 3,0 \\
\hline Sem informação & 13 & 18,6 & & 147 & 10,9 & & 160 & 11,3 & \\
\hline \multicolumn{10}{|l|}{ Hábito alimentar } \\
\hline Sangue humano*** & 24 & 34,3 & 53,3 & 416 & 30,9 & 42,4 & 440 & 31,1 & 42,9 \\
\hline Outros ${ }^{\star \star \star \star}$ & 7 & 10,0 & 15,6 & 154 & 11,4 & 15,7 & 161 & 11,4 & 15,7 \\
\hline Não reagente & 14 & 20,0 & 31,1 & 411 & 30,6 & 41,9 & 425 & 30,0 & 41,4 \\
\hline Sem informação & 25 & 35,7 & & 364 & 27,1 & & 389 & 27,5 & \\
\hline
\end{tabular}

* Percentual calculado excluindo-se as perdas de informação.

** T. = Triatoma; P. = Panstrongylus; R. = Rhodnius

*** Reagente para sangue humano sozinho ou associado a outros.

$\star \star \star \star$ Outros: reagentes para sangue de ave, cão, gato, roedor ou marsupial.

Instituição. Como já observado no período entre 1984-1997 (Carvalho, 2000), na região do planalto paulista, representada pelos Serviços Regionais 04 a 11 da SUCEN (Tabela 3), a maioria dos casos sororreagentes provavelmente autóctones detectados no PCDCh, estaria associada à aquisição da infecção em áreas sabidamente endêmicas para doença de Chagas no estado, até fins dos anos 1960. Torna-se difícil admiti-los como sendo de transmissão recente, ademais do fato de não ter sido incluída, como norma no PCDCh, a colheita de duas amostras de sangue com intervalo suficiente para possibilitar a observação de possíveis soro-conversões. Esse modelo, adotado com sucesso em áreas endêmicas para doença de Chagas por Puigbó et al. (1969) na Venezuela, e por Dias (1974) em Bambuí, Minas Gerais, Brasil, revelou taxas de conversão sorológica de $16,3 \%$ e de $0,4 \%$, respectivamente. Tal desafio deverá ser também incluído nas atividades de sorologia em São Paulo. 
A ausência de sororreagentes observada nas idades inferiores a dez anos pode fazer-nos supor que não houve ocorrência de transmissão congênita nessa faixa etária da amostra examinada. Entretanto, como o grupo de mulheres em idade fértil (15 a 49 anos) incluiu um número expressivo de resultados sororreagentes no total de positivos (Tabela 2), poder-se-ia recomendar o seguimento sorológico das proles dessas mulheres. E seria esse um importante uso da sorologia nas condições atuais de controle em que se encontra o Estado de São Paulo.

Em decorrência da metodologia preconizada para o uso da sorologia no PCDCh, o trabalho incluiu uma amostra de UDs com presença de triatomíneos vetores, não necessariamente representativa das localidades ou dos municípios onde se encontravam. Mesmo assim, os resultados indicaram que a distribuição de reagentes sorológicos deu-se ao acaso, sugerindo que esse instrumento deva ser redirecionado para auxiliar o estudo das repercussões da presença de vetores em domicílios humanos.

O PCDCh no Estado de São Paulo tem por base a vigilância entomológica e esta, a partir de análises acuradas dos encontros de vetores, poderia, de acordo com os moldes anteriormente desenvolvidos, desencadear o uso da sorologia em situações mais específicas do que as até então apresentadas. Condições como a de encontro de um único exemplar de triatomíneo seriam substituídas pela de encontro de colônias de vetores nos domicílios, com infestações intra e peridomicílios e com instalação, no intradomicílio, de vetores infectados por Tr. cruzi. A presença de $P$. megistus e de T. sordida no domicílio, com morador sororreagente para Tr. cruzi, poderia merecer certa atenção por causa dos possíveis riscos relacionados com essas espécies, como já assinalados por Wanderley (1993). Entretanto, neste trabalho, foram pouco expressivos os sinais de domiciliação, de acordo com os dados observados de freqüências de encontro de ninfas, isoladas ou associadas a adultos, infectadas ou não, bem inferiores às de exemplares adultos. O pronto atendimento a notificações de triatomíneos parece ter confirmado a expectativa vigente de obstaculizar sua instalação no ambiente domiciliar (Silva et al., 1999), tendo em vista ainda que o local de captura predominante, em decorrência da imposição das normas do Programa, foi o interno. Ao redire-

\begin{tabular}{|c|c|c|c|}
\hline \multicolumn{4}{|c|}{$\begin{array}{l}\text { Programa de Controle da Doença de Chagas (PCDCh): encontro de reagentes } \\
\text { e de não reagentes sorológicos em Unidades Domiciliares (UDs), com e sem } \\
\text { presença de triatomíneo infectado, em municípios do Estado de São Paulo, Brasil, } \\
1990 \text { a } 1999 .\end{array}$} \\
\hline \multirow[t]{2}{*}{ Característica } & \multicolumn{2}{|c|}{ UD com presença de morador } & \multirow[t]{2}{*}{ Total } \\
\hline & Sororreagente & Negativo & \\
\hline \multicolumn{4}{|l|}{ UD com triatomíneo } \\
\hline infectado por Tr. cruzi & 23 & 363 & 386 \\
\hline não infectado por Tr. cruzi & 36 & 851 & 887 \\
\hline Total & 59 & 1.214 & 1.273 \\
\hline
\end{tabular}

Odds ratio $=1,498(0,875<\mathrm{OR}<2,564,95 \%$ de confiança) $; \mathrm{p}=0,1473$.

cionar a sorologia, deveriam ainda ser consideradas como situações com potencialidade de risco, as representadas por áreas com presença do principal vetor do estado em épocas anteriores, T. infestans, sob quaisquer condições de reintrodução, bem sucedida ou não. Inquéritos sorológicos populacionais poderiam ser desencadeados em situações que viessem a representar risco de transmissão, envolvendo grupos migratórios procedentes de áreas endêmicas para a doença de Chagas, instalados em assentamentos de ocupação recente, com a presença de triatomíneos vetores, mesmo os considerados secundários.

Existem ainda, no Estado de São Paulo, áreas de biocenose silvestre para a doença de Chagas. A sorologia poderia auxiliar na identificação e estudo mais pormenorizado de casos em que isolados de Tr. cruzi pudessem ser analisados quanto à relação parasita-hospedeiro. Deveriam ser então usadas técnicas de aquisição recente (Diotaiuti et al., 1995; Fernandes et al., 1997; Zingales et al., 1998), para possibilitar a identificação de possíveis elos epidemiológicos entre cepas silvestres e domésticas de Tr. cruzi. Embora à SUCEN caiba a investigação de casos agudos, visando a sua classificação quanto ao modo de transmissão, aqueles possivelmente crônicos detectados por mais de uma técnica sorológica, como imunofluorescência indireta e imunoensaio enzimático, por exemplo, devem ser encaminhados a Centros de Referência identificados previamente em nosso estado. 
Referências

ALBARRACIN-VEIZAGA, H.; CARVALHO, M. E.; NASCIMENTO, E. M. M.; RODRIGUES, V. L. C. C.; CASANOVA, C. \& BARATA, J. M. S., 1999. Chagas disease in an area of recent occupation in Cochabamba, Bolivia. Revista de Saúde Pública, 33:201-207.

ANDRADE, A. L. S. S., 1994. Avaliação de Possíveis Fatores de Risco para a Infecção pelo Trypanosoma cruzi em Crianças - Estudo Caso-Controle em Área Rural do Estado de Goiás. Tese de doutorado, São Paulo: Faculdade de Saúde Pública, Universidade de São Paulo.

ANDRADE, A. L. S. S.; ZICKER, F.; LUQUETTI, A. O.; OLIVEIRA, R. M.; SILVA, A. S.; SOUZA, J. M. P. \& MARTELLI, C. M. T., 1992. Surveillance of Trypanosoma cruzi transmission by serological screening of schoolchildren. Bulletin of the World Health Organization, 70:625-629.

BURALLI, G. M., 1985. Estudo do Controle dos Triatomíneos Domiciliados no Estado de São Paulo. Dissertação de Mestrado, São Paulo: Faculdade de Saúde Pública, Universidade de São Paulo.

CARVALHO, M. E., 2000. Sorologia da Infecção Chagásica no Programa de Controle do Estado de São Paulo, Brasil. Tese de Doutorado, São Paulo: Faculdade de Saúde Pública, Universidade de São Paulo.

CARVALHO, M. E.; WANDERLEY, D. M. V. \& ALMEIDA, M. C. R. R., 1987. Sorologia da infecção chagásica em áreas restritas de focos de triatomíneos vetores no Estado de São Paulo. Revista da Sociedade Brasileira de Medicina Tropical, 20(Sup. 2):15.

CARVALHO, M. E.; ZANARDI, A.; DELGADO, M. R.; YAZAWA, R. Y.; MATOS, G. F. \& WANDERLEY, D. M. V., 1993/1994. Infecção chagásica em moradores de unidades domiciliares com presença de triatomíneos vetores no Estado de São Paulo, 1984-1993. Revista de Ciências Farmacêuticas, 15(Sup.):16.

CHUIT, R.; SUBIAS, E.; PEREZ, A. C.; PAULONE, I.; WISNIVESKY-COLLI, C. \& SEGURA, E. L., 1989. Usefulness of serology for the evaluation of Trypanosoma cruzi transmission in endemic areas of Chagas' disease. Revista da Sociedade Brasileira de Medicina Tropical, 22:119-124.

COSTA, F. D. A.; FONSECA, J. A. B.; CARVALHO, M. E.; WANDERLEY, D. M. V.; CIARAVOLO, R. M. C.; MASSUCATO, M. A. S. \& GODO, C., 1982. Resultados preliminares de inquérito soroepidemiológico de doença de Chagas no Litoral Sul do Estado de São Paulo. In: 7o Congresso da Sociedade Brasileira de Parasitologia, Resumos, p. 149. Porto Alegre: Sociedade Brasileira de Parasitologia.

COUTINHO, J. O., 1962. Contribuição ao estudo da epidemiologia da doença de Chagas. Arquivos de Higiene e Saúde Pública, 27:317-330.

DAWSON-SAUNDERS, B. \& TRAPP, R. G., 1994. Basic \& Clinical Biostatistics. 2nd Ed. Norwalk: Appleton \& Lange.

DEAN, A. G.; DEAN, J. A.; COULOMBIER, D.; BRENDEL, K. A.; SMITH, D. C.; BURTON, A. H.; DICKER, R. C.; SULLIVAN, K.; FAGAN, R. F. \& ARNER, T. G., 1994. Epi Info, Version 6: A Word Processing Database, and Statistics Program for Epidemiology on Microcomputers. Atlanta: Centers for Disease Control and Prevention.
DIAS, J. C. P., 1967. Prevalência da doença de Chagas em crianças da zona rural de Bambuí, MG, após ensaio profilático. Revista Brasileira de Malariologia, 29:135-159.

DIAS, J. C. P., 1974. Perspectivas para o Controle da Doença de Chagas Humana em Áreas Endêmicas Através de Profilaxia Domiciliar com Inseticidas de Ação Residual. Experiência de Bambuí, Minas Gerais, Brasil. Dissertação de Mestrado, Belo Horizonte: Faculdade de Medicina, Universidade Federal de Minas Gerais.

DIOTAIUTI, L.; PEREIRA, A. S.; LOIOLA, C. F; FERNANDES, A. J.; SCHOFIELD, J. C.; DUJARDIN, J. P; DIAS, J. C. P. \& CHIARI, E., 1995. Inter-relation of sylvatic and domestic transmission of Trypanosoma cruzi in areas with and without domestic vectorial transmission in Minas Gerais, Brazil. $\mathrm{Me}$ mórias do Instituto Oswaldo Cruz, 90:443-448.

FERNANDES, C. D.; MURTA, S. M. F.; CERÁVALO, I. P.; KRUG, L. P.; VIDIGAL, P. G.; STEINDEL, M.; NARDI, N. \& ROMANHA, A. J., 1997. Characterization of Trypanosoma cruzi strains from chronic chagasic patients, triatomines and opossums naturally infected from the State of Rio Grande do Sul, Brazil. Memórias do Instituto Oswaldo Cruz, 92:343-351.

GOLDSMITH, R. S.; ZARATE, R. J.; ZARATE, L. G.; KAGAN, I. G. \& JACOBSON, L. B., 1985. Clinical and epidemiological studies of Chagas' disease in rural communities in Oaxaca State, Mexico, and a seven-year follow-up: I. Pan American Health Organization Bulletin, 19:120-138.

GUARITA, O. F.; FOMM, A. S.; BRIGIDO, R. M. \& PIMENTA FILHO, T. T., 1978. Inquérito Sorológico para Avaliação da Infecção Chagásica do Grupo Etário de 9 a 14 Anos dos Escolares do Estado de São Paulo, Brasil. São Paulo: Secretaria de Estado da Saúde de São Paulo.

HOFF, R.; TODD, C. W.; MAGUIRE, J. H.; PIESMAN, J.; MOTT, K. E.; MOTA, E. E.; SLEIGH, A.; SHERLOCK, I. A. \& WELLER, T. H., 1985. Serologic surveillance of Chagas' disease. Annales de la Societé Belge de Médecine Tropicale, 65(Sup. 1):187-196.

LEITE, O. F; ALVES, M. J. C. P.; SOUZA, S. S. L.; MAYO, R. C.; ANDRADE, V. R.; SOUZA, C. E.; RANGEL, O.; OLIVEIRA, S. S.; LIMA, V. L. C.; RODRIGUES, V. L. C. C.; CARVALHO, M. E.; CASANOVA, C. \& WANDERLEY, D. M. V., 2001. Triatoma infestans em área sob vigilância entomológica para doença de Chagas, Estado de São Paulo, Brasil. Revista da Sociedade Brasileira de Medicina Tropical, 34:437-443.

PIESMAN, J.; MOTA, E.; SHERLOCK, I. A. \& TODD, C. W., 1985. Trypanosoma cruzi: Association between seroreactivity of children and infection rates in domestic Panstrongylus megistus (Hemiptera: Reduviidae). Journal of Medical Entomology, 22: 130-133.

PUIGBÓ, J. J.; NAVA, J. R. R.; GARCIA, H. B \& GIL, C. Y., 1969. Cuatro años de estudio longitudinal de uma comunidad rural com endemicidad chagasica. Boletín de la Oficina Sanitaria Panamericana, 66:112-120.

ROCHA-E-SILVA, E. O.; GUARITA, O. F. \& ISHIHATA, G. K., 1979. Doença de Chagas: Atividades de contro- 
le dos transmissores no Estado de São Paulo; Brasil. Revista Brasileira de Malariologia, 31:99-119.

SALVATELLA, R.; CALEGARI, L.; CASSERONE, S.; CIVILA, E.; CARBAJAL, S.; PEREZ, G.; SOMMA, R.; SAMPAIO, I.; LLANES, M. E.; CONTI, M.; PAULERCI, C.; CONTI-DIAZ, I. A.; SOMMA-MOREIRA, R. \& PEREZ MOREIRA, L., 1989. Seroprevalencia de anticuerpos contra Trypanosoma cruzi en 13 Departamentos del Uruguay. Boletín de la Oficina Sanitaria Panamericana, 107:108-117.

SILVA, R. A.; BONIFÁCIO, P. R. \& WANDERLEY, D. M. V., 1999. Doença de Chagas no Estado de São Paulo: Comparação entre pesquisa ativa de triatomíneos em domicílios e notificação de sua presença pela população em área sob vigilância entomológica. Revista da Sociedade Brasileira de Medicina Tropical, 32:653-659.

SOLIS-ACOSTA, H. M.; FERREIRA, C. S. \& CARVALHO, M. E., 1997. Seroepidemiology of Chagas' disease in Nasca, Southern region of Peru. Revista do Instituto de Medicina Tropical de São Paulo, 39:107-112.

VELASCO-HERNANDEZ, J. X., 1991. An epidemiological model for the dynamics of Chagas' disease. BioSystems, 26:127-134.

VELASCO-CASTREJON, O.; VALDESPINO, J. L.; TAPIACONYER, R.; SALVATIERRA, B.; GUZMAN-BRACHO, C.; MAGOS, C.; LLAUSAS, A.; GUTIERREZ, G. \& SEPULVEDA, J., 1992. Seroepidemiología de la enfermedad de Chagas en México. Salud Pública de México, 34:186-196.

WANDERLEY, D. M. V., 1993. Vigilância do risco de transmissão da doença de Chagas por vetores secundários no Estado de São Paulo. Revista da Sociedade Brasileira de Medicina Tropical, 26(Sup. 2):38-40.

ZINGALES, B.; SOUTO, R. P.; MANGIA, R. H.; LISBOA, C. V.; CAMPBELL, D. A.; COURA, J. R.; JANSEN, A. \& FERNANDES, O., 1998. Molecular epidemiology of American trypanosomiasis in Brazil based on dimorphisms of RNA and mini-exon gene sequences. International Journal of Parasitology, 28:105-112.

Submetido em 27 de agosto de 2001

Versão final reapresentada em 19 de março de 2002

Recebido em 17 de maio de 2002 